\title{
Prognostic Factors for Patients With a Large Number of Hepatocellular Carcinoma Nodules
}

\author{
Yutaro Abe ${ }^{a}$, Kazuyoshi Ohkawa ${ }^{a}$ e, Mitsuru Sakakibara ${ }^{a}$, Takatoshi Nawa ${ }^{\text {a }}$, \\ Nobuyasu Fukutakea , Kenji Ikezawa ${ }^{\text {a }}$, Ryoji Takada ${ }^{a}$, Akira Kusakabea, \\ Toshihiro Imai ${ }^{\mathrm{a}}$, Katsuyuki Nakanishi ${ }^{\mathrm{b}}$, Takahiro Tabuchic, Hayato Hikita ${ }^{\mathrm{d}}$, \\ Ryotaro Sakamori $^{\mathrm{d}}$, Tomohide Tatsumi ${ }^{\mathrm{d}}$, Tetsuo Takehara ${ }^{\mathrm{d}}$, Kazuhiro Katayama ${ }^{\mathrm{a}}$
}

\begin{abstract}
Background: The prognostic factors and treatment strategies for hepatocellular carcinoma (HCC) patients with a large number of tumor nodules have not been fully elucidated. Clinical factors influencing prognosis were investigated in HCC patients with 30 or more tumor nodules.
\end{abstract}

Methods: Forty-six HCC patients with 30 or more tumor nodules participated in this study. None of them had vascular invasion and extrahepatic metastasis. Kaplan-Meier curve and Cox proportional hazard model were used for analysis.

Results: The median survival time of our patients was no more than 15 months, suggesting that patients with 30 or more tumor nodules may be regarded as a progressive subgroup showing poorer prognosis. In multivariate analysis, presence of between 30 and 59 tumor nodules $(P=0.002)$, male gender $(P=0.002)$, lower total bilirubin (total bilirubin $<1.0 \mathrm{mg} / \mathrm{dL})(\mathrm{P}=0.011)$, transarterial chemoembolization (TACE) as an initial therapy $(\mathrm{P}=0.027)$ and higher prothrombin time $(\mathrm{P}=0.049)$ were significant independent factors for better overall survival. Among 39 patients who underwent TACE as an initial therapy, patients who received sorafenib therapy during follow-up showed better overall survival than those who did not $(\mathrm{P}=0.026)$. Efficacy of sorafenib appeared to be more evident in patients who needed repeated transarterial treatment.

Conclusions: In HCC patients with 30 or more tumor nodules, TACE as an initial therapy may be correlated with better prognosis.

Manuscript submitted November 21, 2019, accepted December 9, 2019

aDepartment of Hepatobiliary and Pancreatic Oncology, Osaka International Cancer Institute, 3-1-69 Otemae, Chuo-ku, Osaka 541-8567, Japan

bDepartment of Diagnostic Radiology, Osaka International Cancer Institute, 3-1-69 Otemae, Chuo-ku, Osaka 541-8567, Japan

${ }^{\mathrm{c}}$ Department of Cancer Epidemiology, Osaka International Cancer Institute, 3-1-69 Otemae, Chuo-ku, Osaka 541-8567, Japan

dDepartment of Gastroenterology and Hepatology, Osaka University Graduate School of Medicine, 2-2 Yamadaoka, Suita,Osaka 565-0871, Japan

eCorresponding Author: Kazuyoshi Ohkawa, Department of Hepatobiliary and Pancreatic Oncology, Osaka International Cancer Institute, 3-1-69 Otemae, Chuo-ku, Osaka 541-8567, Japan. Email: ohkawa-ka@mc.pref.osaka.jp

doi: https://doi.org/10.14740/jocmr4032
Sorafenib administration after the prior transarterial treatment may improve antitumor efficacy.

Keywords: A large number of hepatocellular carcinoma nodules; Overall survival; Transarterial chemoembolization; Sorafenib

\section{Introduction}

Hepatocellular carcinoma (HCC) is one of the most lethal and prevalent cancers. It is the sixth most common cancer and third most common cause of cancer-related death worldwide [1]. The prognosis and treatment of patients with HCC are determined by tumor burden, liver function reserve and general health status including comorbidities [2-5]. In HCC, massive tumor expansion, invasion to major intrahepatic vessels and extrahepatic metastasis are known to be factors associated with poor prognosis [2-5]. Without these factors, however, experience shows that patients having an extremely large number of HCC nodules of modest size reveal poor prognosis as well. Such tumor status belongs to the intermediate stage (Barcelona clinic liver cancer (BCLC) stage B), that includes patients having Child-Pugh A or B liver function with four or more tumors irrespective of size or $2-3$ tumors larger than $3 \mathrm{~cm}$ in maximum diameter in the absence of cancer-related symptoms, macrovascular invasion, or extrahepatic spread [2, 4-6]. Classification of the intermediate stage of HCC into substages has been attempted because this stage comprises a widely variable patient population in the tumor burden and liver function [79]. For example, subgrouping system of the intermediate stage into B1 to B4 groups based on the up-to-seven criteria has been proposed by an expert panel [7] and further subgrouping systems have been validated by a few investigators $[10,11]$. However, the tumor status of extremely large number of HCC nodules that was focused on in the present study is far from these subgrouping systems and expresses the progressive disease stage. Although transarterial chemoembolization (TACE) is the recommended treatment for intermediate-stage $\mathrm{HCC}[2$, 4-6], the optimal treatment strategies for HCC patients with a large number of tumor nodules have not been fully elucidated.

To better address this, we carried out the retrospective 
analysis of HCC patients with a large number of tumor nodules. In this study, "a large number of tumor nodules" was defined expedientially as tumor nodules of 30 or more. The prognostic factors and appropriate treatment for these patients were investigated in this study.

\section{Patients and Methods}

\section{Patients}

Forty-six patients were selected among 507 patients who underwent hepatic angiographies in Osaka Medical Center for Cancer and Cardiovascular Diseases (renamed International Cancer Institute in March 2017) between April 2010 and February 2015. Inclusion criteria are as follows: 1) tumor status of patient belonging to the intermediate stage (BCLC stage B); 2) patients diagnosed as having liver tumor nodules 30 or more which were confirmed as HCC by angiography; and 3) patients received any aggressive anticancer treatment. This study was designed in keeping with the Declaration of Helsinki and approved by Ethics Committee of Osaka International Cancer Institute and acceptance number was 1611079161.

\section{Diagnosis of $\mathrm{HCC}$}

First, HCC was diagnosed using contrast-enhanced computed tomography (CECT), gadolinium-ethoxybenzyl-diethylenetriamine pentaacetic acid-enhanced magnetic resonance imaging (MRI) or contrast-enhanced ultrasound. HCC was confirmed by angiography based on the enhancement in the arterial phase and the washout in the portal phase. Angiography was performed through the femoral artery. CT during arterial portography (CTAP) was conducted from superior mesenteric artery by infusion of contrast material. Digital subtraction angiography was done from the celiac artery to evaluate hepatic arterial and tumor feeding arterial anatomy. CT during hepatic arteriography (CTHA) was evaluated by injecting contrast material through the common hepatic artery, proper hepatic artery and feeding artery of the targeted tumor. HCC was diagnosed when a tumor was detected as a perfusion defect at CTAP and arterial enhancement at CTHA. The number of nodules was counted carefully based on the CTHA and CTAP findings.

\section{Treatment}

Each treatment was administered on the basis of the tumor burden, liver function and general health status according to the judgment of each attending physician. TACE was conducted using anticancer drugs, poppy-seed oil (Lipiodol, Gueret Japan, Tokyo, Japan) and gelatin particles. Chemoembolization was performed using epirubicin (Nippon Kayaku, Tokyo, Japan) or cisplatin (IA call, Nippon Kayaku) via tumor feeding artery. The injection volume of emulsion and embolic material was determined according to tumor size, tumor number and liver function. As for transarterial infusion chemotherapy
(TAI), $65 \mathrm{mg} / \mathrm{m}^{2}$ cisplatin (IA call, Nippon Kayaku) was injected by using automatic injection machine for $30 \mathrm{~min}$ from the right or left hepatic artery or both. Sorafenib (Nexavar; Bayer Pharmaceuticals, Osaka, Japan) treatment was started at an initial dose of 400 to $800 \mathrm{mg} /$ day. Dosage was reduced or discontinued according to the degree of toxicity. Patients, in whom the duration of the sorafenib treatment was less than 2 weeks, were regarded to be unadministered.

\section{Follow-up}

Clinical, laboratory and radiological assessments were performed to evaluate tumor status. Recurrence was treated with TACE, TAI or sorafenib as appropriate. Overall survival was measured from the date of the first day when tumor number has reached 30 nodules or more until the date of death.

\section{Statistical analysis}

Univariate and multivariate analyses with Cox proportional hazard model were used to determine the prognostic factors. Hazard ratio (HR) and 95\% confidence interval (CI) were obtained. Cumulative survival rates were analyzed by the Kaplan-Meier method. The differences between survival curves were compared by the generalized Wilcoxon test [12] in order to give statistical weight to the patients in which long time has not been passed since an initial treatment. The two groups were compared by Fisher's exact tests and Mann-Whitney non-parametric U-tests. $\mathrm{P}<0.05$ was considered to be statistically significant. Statistical analysis was performed using SPSS version 21.0 (IBM, Chicago, IL, USA).

\section{Results}

\section{Patient clinical feature}

Of the 46 patients, there were 32 men and 14 women, with a median age of 73 (range, 58 - 88) years. The etiologies of liver diseases included hepatitis $\mathrm{C}$ (as determined by positive hepatitis $\mathrm{C}$ virus (HCV) antibody) in $33(72 \%)$ patients, hepatitis $\mathrm{B}$ (as determined by positive hepatitis B virus (HBV) surface antigen) in six (13\%) patients and neither hepatitis $\mathrm{B}$ or $\mathrm{C}$ in seven $(15 \%)$ patients. Among hepatitis $\mathrm{C}$ patients, antiviral treatment with interferon alone or combination with ribavirin was carried out in eight patients, one of whom had achieved the sustained eradication of the virus. Among hepatitis B patients, the sustained negative HBV DNA after antiviral treatment with entecavir was achieved in four patients. The median alanine aminotransferase (ALT) and total bilirubin levels were $37 \mathrm{U} / \mathrm{L}$ (range, $14-101 \mathrm{U} / \mathrm{L}$ ) and $0.9 \mathrm{mg} / \mathrm{dL}$ (range, 0.5 - 1.9 $\mathrm{mg} / \mathrm{dL}$ ). The median prothrombin time was $81 \%$ (range, 58 - 104\%). Nine (20\%) patients were habitual drinkers of $>60$ $\mathrm{mg}$ /day in ethanol equivalent. Of the 46 patients, $34(74 \%)$ patients were classified as Child-Pugh A, 11 (24\%) were as B and one $(2 \%)$ was classified as $\mathrm{C}$. The median of maximum 
Table 1. Previous Treatment, Initial Treatment and After Treatment of the 46 HCC Patients With 30 Nodules or More

\begin{tabular}{|cl|}
\hline Content of therapy & No. (\%) \\
\hline Previous treatment & $43(93 \%)$ \\
\hline Any & $38(83 \%)$ \\
\hline TACE & $3(7 \%)$ \\
\hline TAI & $22(48 \%)$ \\
\hline RFA & $13(28 \%)$ \\
\hline PEI & $14(30 \%)$ \\
\hline Hepatic resection & $46(100 \%)$ \\
\hline Initial treatment & $39(85 \%)$ \\
\hline Any & $7(15 \%)$ \\
\hline TACE & $1(2 \%)$ \\
\hline TAI & \\
\hline Sorafenib & $35(76 \%)$ \\
\hline After treatment & $26(57 \%)$ \\
\hline Any & $17(37 \%)$ \\
\hline TACE & $11(24 \%)$ \\
\hline TAI & \\
\hline Sorafenib & \\
\hline
\end{tabular}

HCC: hepatocellular carcinoma; TACE: transarterial chemoembolization; TAI: transarterial infusion chemotherapy; RFA: radiofrequency ablation; PEI: percutaneous ethanol injection.

diameter of tumor was $26 \mathrm{~mm}$ (range, 13 - $60 \mathrm{~mm}$ ). As for the tumor number, 38 (83\%) patients had 30 - 59 HCC nodules and $16(17 \%)$ patients had $\geq 60 \mathrm{HCC}$ nodules. The median AFP and DCP levels were $55 \mathrm{ng} / \mathrm{mL}$ (range, $<5-36,927 \mathrm{ng} / \mathrm{mL}$ ) and $188 \mathrm{mAU} / \mathrm{mL}$ (range, $<30-38,000 \mathrm{mAU} / \mathrm{mL}$ ). Patients were followed up at a median period of 12 months (range, 1 70 months). Thirty-nine patients (85\%) died during follow-up.

Previous treatment, initial treatment and after treatment of the 46 HCC patients with 30 nodules or more are shown in Table 1. In the present study, "initial treatment" meant "the treatment just after the tumor number has reached 30 or more". Forty-three $(93 \%)$ of the $46 \mathrm{HCC}$ patients had received the previous treatment, TACE in $38(83 \%)$ patients, TAI in three (7\%) patients, radiofrequency ablation (RFA) in $22(48 \%)$ patients, percutaneous ethanol injection (PEI) in 13 (28\%) patients and hepatic resection in $14(30 \%)$ patients. As for the initial treatment, 39 (85\%) patients underwent TACE, seven $(15 \%)$ patients underwent TAI and one $(2 \%)$ patient underwent sorafenib administration. After treatment was given in 35 (76\%) patients, TACE in $26(57 \%)$ patients, TAI in $17(37 \%)$ patients and sorafenib administration in 11 (24\%) patients.

\section{Factors contributing to overall survival}

Cumulative overall survival of the $46 \mathrm{HCC}$ patients with 30 nodules or more was first analyzed by Kaplan-Meier curve (Fig. 1). The survival rates at 1, 2 and 3 years were 55\%, 22\%

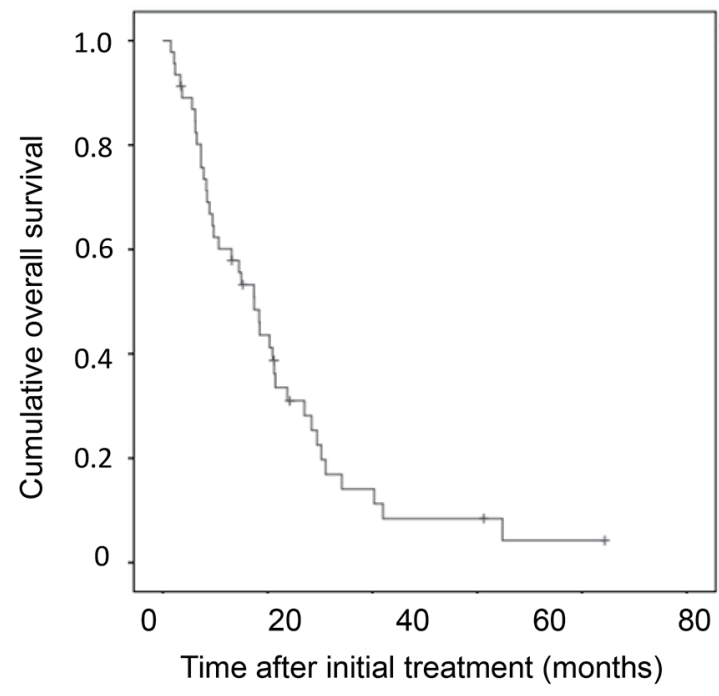

$\begin{array}{llllll}\text { No. at risk } & 46 & 12 & 3 & 1 & 0\end{array}$

Figure 1. Kaplan-Meier analysis of cumulative OS of the $46 \mathrm{HCC}$ patients with 30 nodules or more. OS: overall survival; HCC: hepatocellular carcinoma.

and $1 \%$. The median survival time was 15 months. Next, factors contributing to overall survival were studied by using univariate and multivariate analyses (Table 2). Fifteen clinical factors, age, gender, HCV positivity, HBV positivity, amount of drinking ( $<60 \mathrm{vs}$. $\geq 60 \mathrm{mg} /$ day in ethanol equivalent), Child-Pugh classification (A vs. B/C), alanine aminotransferase, total bilirubin, albumin, prothrombin time, $\operatorname{AFP}(<100$ vs. $\geq 100 \mathrm{ng} / \mathrm{mL})$, DCP ( $<200$ vs. $\geq 200 \mathrm{mAU} / \mathrm{mL})$, maximum diameter of tumor, number of tumors (30 - 59 vs. $\geq$ 60 nodules) and initial treatment (TACE or not) were used as variables for univariate analysis. Higher serum albumin (HR, 0.304; 95\% CI, $0.144-0.642 ; \mathrm{P}=0.002$ ), fewer tumors (30 - 59 nodules) $(\mathrm{HR}, 0.378 ; 95 \% \mathrm{CI}, 0.187-0.763 ; \mathrm{P}=$ 0.005 ), higher prothrombin time (HR, 0.965; 95\% CI, 0.940 - 0.991; $\mathrm{P}=0.009)$, TACE as an initial treatment (HR, 0.291; 95\% CI, $0.071-0.762 ; \mathrm{P}=0.012$ ), lower total bilirubin (HR, $0.368 ; 95 \% \mathrm{CI}, 0.161-0.844 ; \mathrm{P}=0.018)$ and male gender (HR, 0.467; 95\% CI, $0.240-0.908$; $\mathrm{P}=0.025$ ) were significant factors associated with better overall survival. Significant factors $(\mathrm{P}<0.05)$ in univariate analysis and age were used in the multivariate analysis, fewer tumors (30 - 59) (HR, 0.284; $95 \%$ CI, $0.130-0.613$; $\mathrm{P}=0.002)$, male gender $(\mathrm{HR}, 0.283$; $95 \%$ CI, $0.130-0.617 ; \mathrm{P}=0.002)$, lower total bilirubin (HR, $0.239 ; 95 \% \mathrm{CI}, 0.080-0.719 ; \mathrm{P}=0.011)$, TACE as an initial treatment $(\mathrm{HR}, 0.301 ; 95 \% \mathrm{CI}, 0.104-0.890 ; \mathrm{P}=0.027)$ and higher prothrombin time (HR, 0.959; 95\% CI, 0.920 - 1.000; $\mathrm{P}=0.049$ ) were selected as significant independent factors for better overall survival. The Kaplan-Meier curves of cumulative overall survival in relation to gender, tumor number, initial treatment (TACE or not), total bilirubin and prothrombin time are shown in Figure 2. Male gender $(\mathrm{P}=0.002)$, tumor number of $30-59$ nodules $(\mathrm{P}=0.002)$, TACE as an initial treatment $(\mathrm{P}=0.033)$, lower total bilirubin $(<1.0 \mathrm{mg} / \mathrm{dL})(\mathrm{P}=$ $0.011)$ and higher prothrombin time $(\mathrm{P}=0.049)$ were associ- 
Table 2. Univariate and Multivariate Analyses to Investigate Factors Contributing to Overall Survival in HCC Patients With 30 Nodules or More

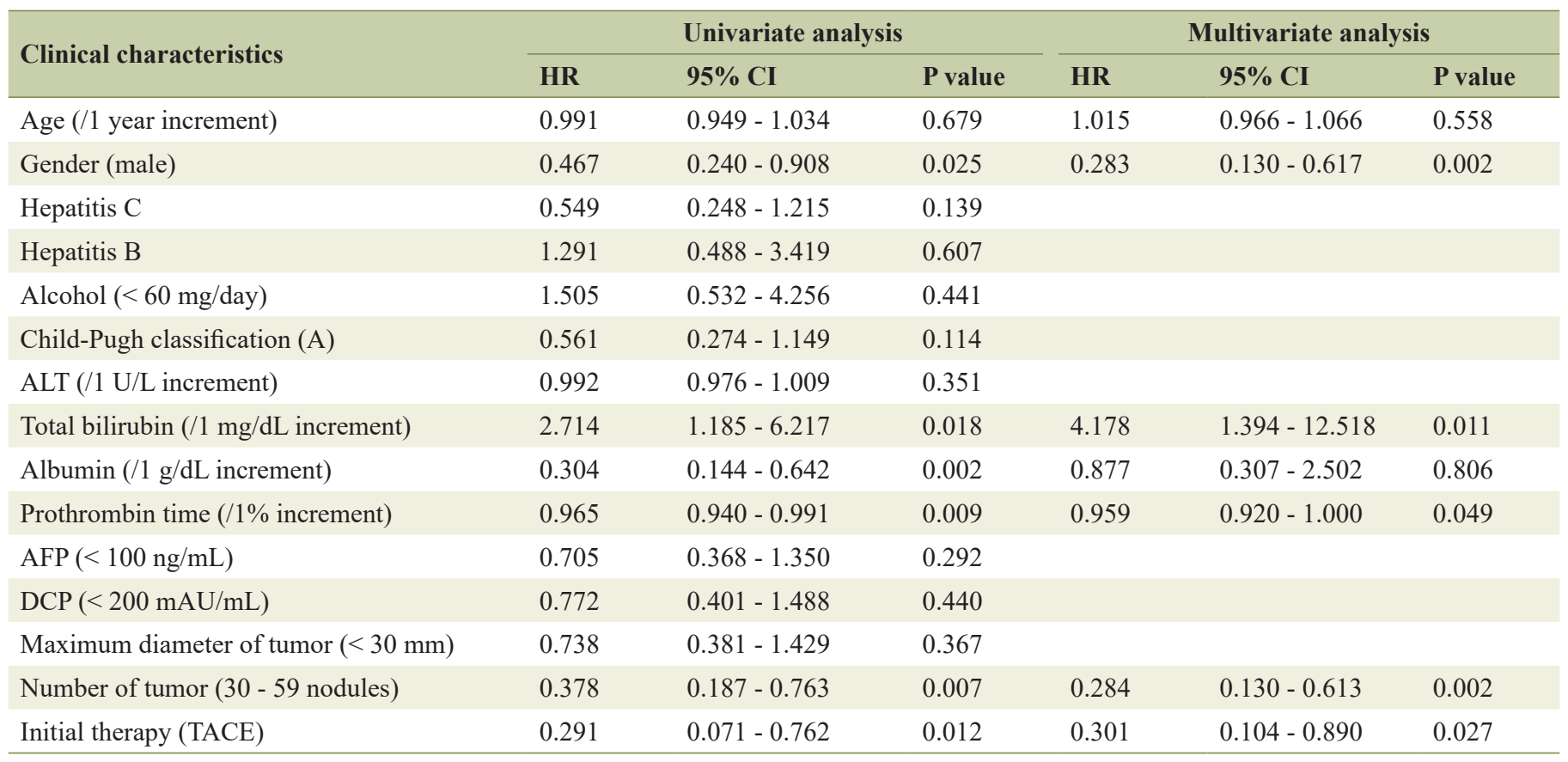

Significant factors $(P<0.05)$ in univariate analysis (gender, total bilirubin, albumin, prothrombin time, number of tumor and initial therapy) and age were used in the multivariate analysis. HCC: hepatocellular carcinoma; HR: hazards ratio; Cl: confidence interval; ALT: alanine aminotransferase; AFP: alpha-fetoprotein; DCP: des-gamma-carboxy prothrombin; TACE: transarterial chemoembolization.

ated with better overall survival.

\section{Association of overall survival of the $39 \mathrm{HCC}$ patients who underwent TACE as an initial treatment}

In $39(85 \%)$ patients who underwent TACE as an initial treatment, subsequent treatment course during the follow-up period is depicted in Figure 3. Of 39 patients, TACE was repeated at least once in $25(64 \%)$ patients. A significantly better overall survival was seen in patients with repeated TACE than in those without it $(\mathrm{P}=0.035)$ (Fig. $4 \mathrm{a})$. TAI was carried out in 14 patients $(36 \%)$ as the after treatment. Overall survival tended to be better in patients who received subsequent TAI than those who did not, although the difference did not reach a statistically significant level $(\mathrm{P}=0.070)$ (Fig. 4b).

Ten $(26 \%)$ patients received sorafenib as after treatment. Their overall survival was better than that of those who did not receive sorafenib $(\mathrm{P}=0.026)$ (Fig. 4c). When the treatment course after initial TACE was followed up in detail in relation to the presence or absence of sorafenib administration as after treatment (Fig. 3), more patients without subsequent sorafenib treatment died in relatively short order compared with those with it. However, several patients who were untreated with sorafenib, and a smaller number of patients treated with sorafenib subsequently, survived for a considerably long period after only once or twice treatments of TACE. In view of the differences in the clinical course between patients with and without subsequent sorafenib treatment, we attempted to investigate in which sub- group of patients sorafenib treatment following TACE would improve the prognosis. As shown in Table 3, no significant differences were observed in the overall survival rate between patients with and without subsequent sorafenib administration in a subgroup of patients who survived for 6 months or more $(\mathrm{n}=$ 32). However, in the subgroup of patients who survived more than 6 months and received TACE or TAI twice or more in addition to the initial TACE $(\mathrm{n}=18)$, a significantly prolonged overall survival was seen in patients who underwent sorafenib treatment compared with those $\operatorname{did} \operatorname{not}(\mathrm{P}=0.033)$. The clinical characteristics were shown in Supplementary Material 1 (www. jocmr.org) and there is no significant statistical difference between patients with and without sorafenib administration. Taken together, in HCC patients with 30 nodules or more in whom the disease was barely controlled by multiple rounds of TACE or TAI, the additional sorafenib administration may confer a substantially favorable antitumor effect.

\section{Discussion}

The prognostic factors and the optimal treatment strategies for HCC patients having a large number of tumor nodules, that is, the most progressive form of intermediate-stage HCC, have not been fully elucidated. To better address this, we attempted to retrospectively investigate patients having an HCC with 30 nodules or more. Because the majority of our patients had previously undergone various medications for HCC, a large number of lesions of them were speculated to be caused by multi- 

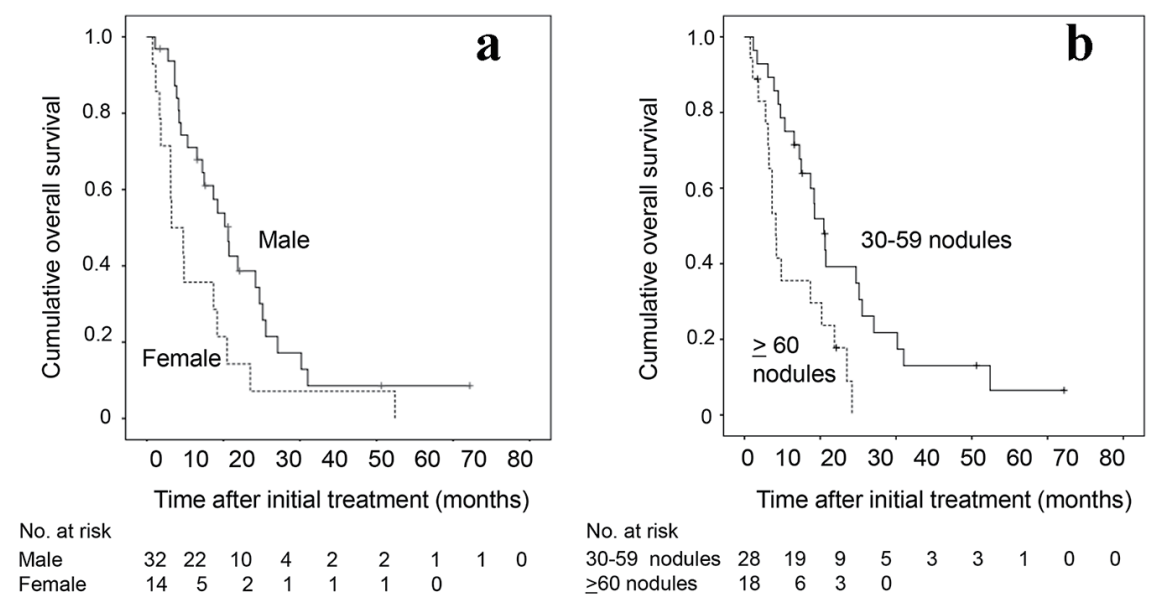

No. at risk
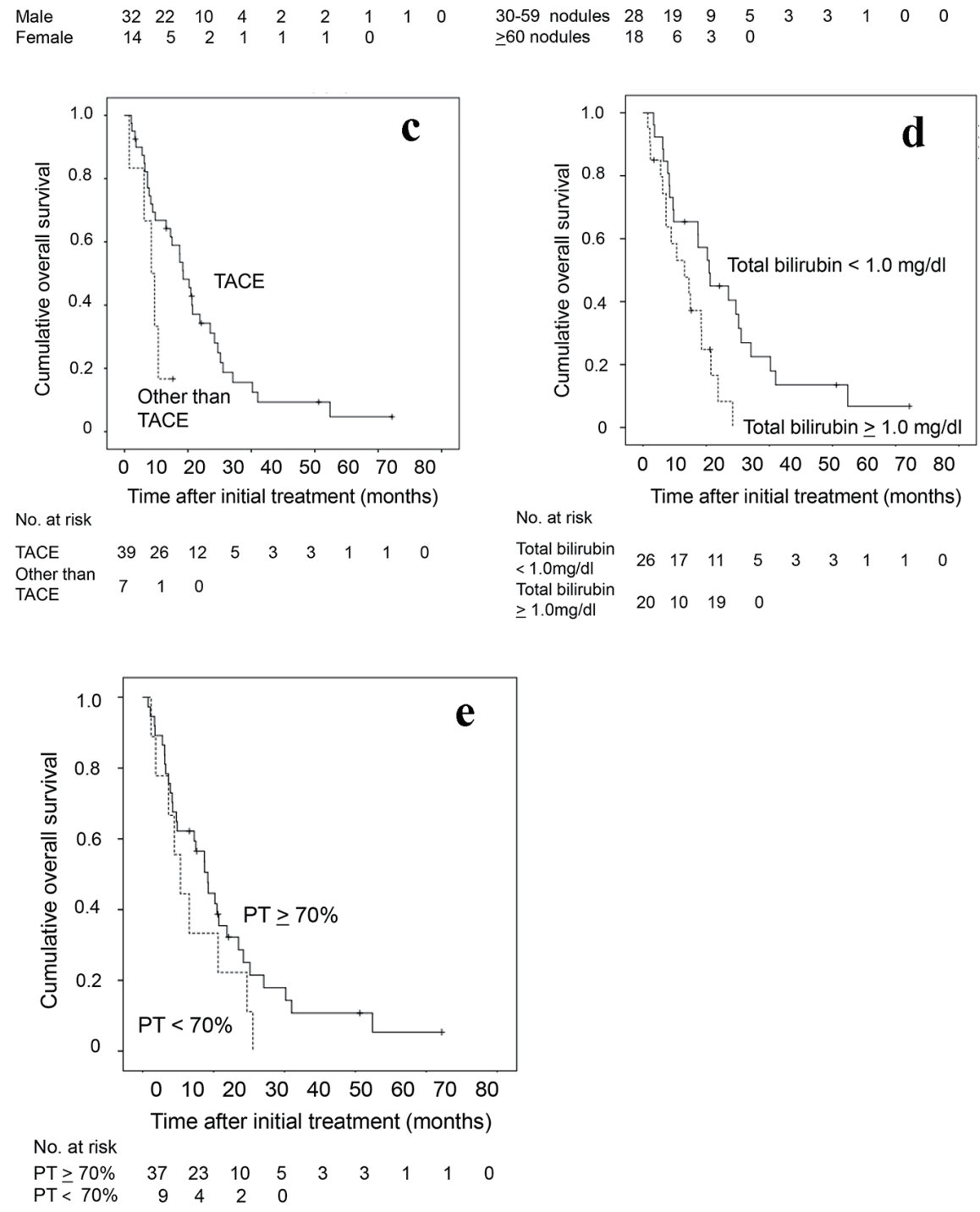

Figure 2. Kaplan-Meier analysis of cumulative OS of the $46 \mathrm{HCC}$ patients with 30 nodules or more in relation to (a) sex, (b) tumor number, (c) initial treatment (TACE or not), (d) total bilirubin and (e) prothrombin time. OS: overall survival; HCC: hepatocellular carcinoma; TACE: transarterial chemoembolization.

ple intrahepatic metastasis from the previously treated primary lesion rather than multicentric carcinogenesis. The median survival time of our patients was only 15 months, which was shorter than patients with intermediate-stage $\mathrm{HCC}$ of less than 


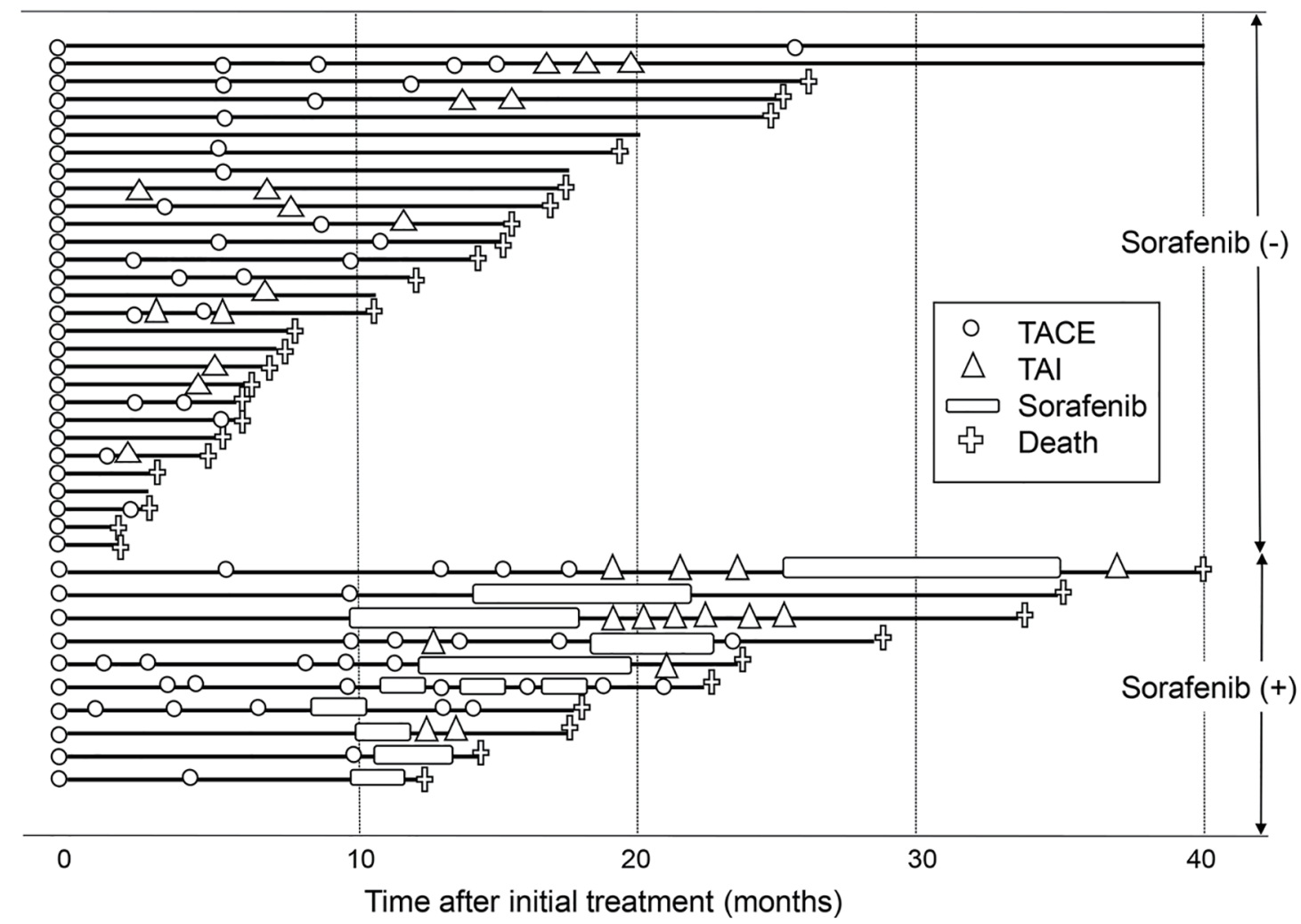

Figure 3. Subsequent treatment course of the 39 patients who underwent TACE as an initial treatment. The solid line denotes the survival time of patients, and the crosses denote deaths. The open circles and open triangles denote TACE and TAI treatments. The open boxes denote sorafenib administration. Patients who received sorafenib are shown in the lower part, whereas patients who did not are shown in the upper part. TACE: transarterial chemoembolization; TAl: transarterial infusion chemotherapy.

30 nodules in our institute [9]. This result indicates that this difficult-to-treat patient group with a large number of HCC nodules would have a poor prognosis, as with advanced stage HCC patients with vascular invasion and/or extrahepatic metastasis. Multivariate analysis revealed that fewer tumors, lower total bilirubin, male gender, TACE as an initial treatment and higher prothrombin time were significantly associated with better overall survival in our patients with a large number of HCC nodules. This suggests that tumor burden and liver function reserve may be main prognostic factors in our patients with a large number of HCC nodules, as is the case for HCC patients with other disease stages [13, 14]. Our study also showed that prognosis was better in males than in females, despite the broad recognition of the tendency toward more progressive disease course in male HCC patients [3]. The small number of patients may have somewhat influenced the result in this study. As for the initial treatment for patients with a large number of HCC nodules, TACE was shown to be the most effective, although our study includes a small number of patients with considerably biased clinical features. For patients in whom TACE was given as the initial treatment, the subsequent treatment was further investigated. Patients who received repeated TACE showed a better prognosis than those who did not. The tendency toward better prognosis was observed in patients who underwent TAI as the after treatment compared with those who did not, although the difference did not reach a statistically significant level. Thus, repetition of transcatheter arterial treatment may contribute to the good prognosis in patients with a large number of HCC nodules. As for the additional treatment of sorafenib, overall survival tended to be longer in patients treated with sorafenib compared with those who were not treated. The clinical course after initial TACE was examined in detail in relation to the additional sorafenib administration. There were a few patients who had considerable long-term survival after once or twice of TACE. Administration of sorafenib did not appear to be needed in these "super-responders to TACE". However, most patients who received sorafenib repeated the transcatheter arterial treatment. Indeed, sorafenib treatment as the after treatment significantly prolonged the overall survival in a subgroup of patients who survived for 6 months or more and repeated TACE or TAI twice or more. Taken together, the efficacy of the combined use of TACE/TAI and sorafenib may be more distinct in patients with a large number of HCC nodules who require repeated TACE/ TAI for tumor control, i.e. "partial-responders to TACE".

Sorafenib is an oral multikinase inhibitor that inhibits vascular endothelial growth factor receptor, platelet-derived growth factor receptor and RAF serine/threonine kinase [15]. Sorafenib is currently recognized as a standard treatment in patients with advanced stage (BCLC stage C) HCC [16-18]. Recently, a 


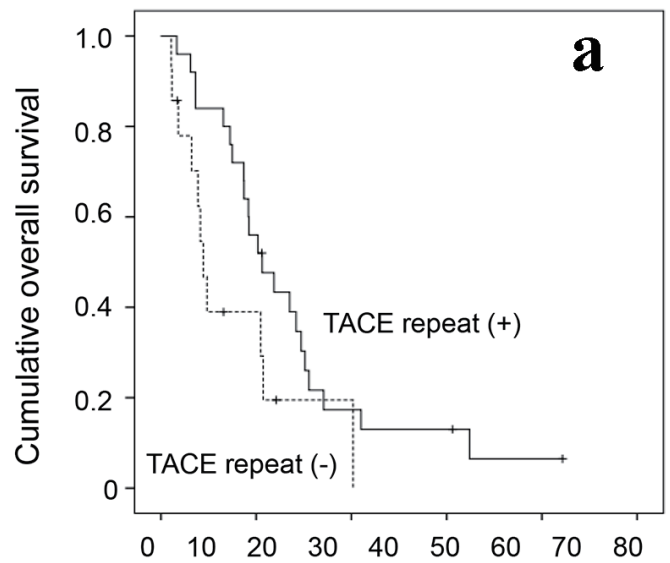

No. at risk

Time after initial treatment (months)

$\begin{array}{llllllllll}\text { TACE repeat (+) } & 25 & 21 & 10 & 4 & 3 & 3 & 1 & 1 & 0\end{array}$

TACE repeat (-) $14 \begin{array}{lllll}5 & 2 & 1 & 0\end{array}$

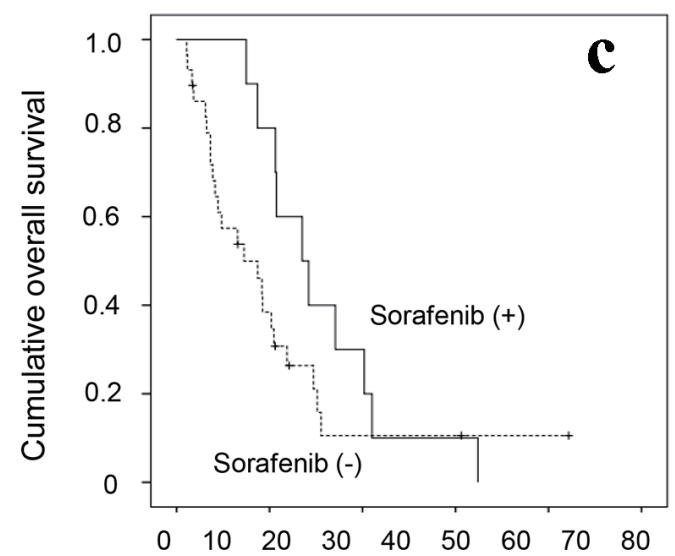

No. at risk

$\begin{array}{llllllll}\text { Sorafenib (+) } & 10 & 9 & 6 & 3 & 1 & 1 & 0\end{array}$

$\begin{array}{llllllllll}\text { Sorafenib (-) } & 29 & 16 & 6 & 2 & 2 & 2 & 1 & 1 & 0\end{array}$

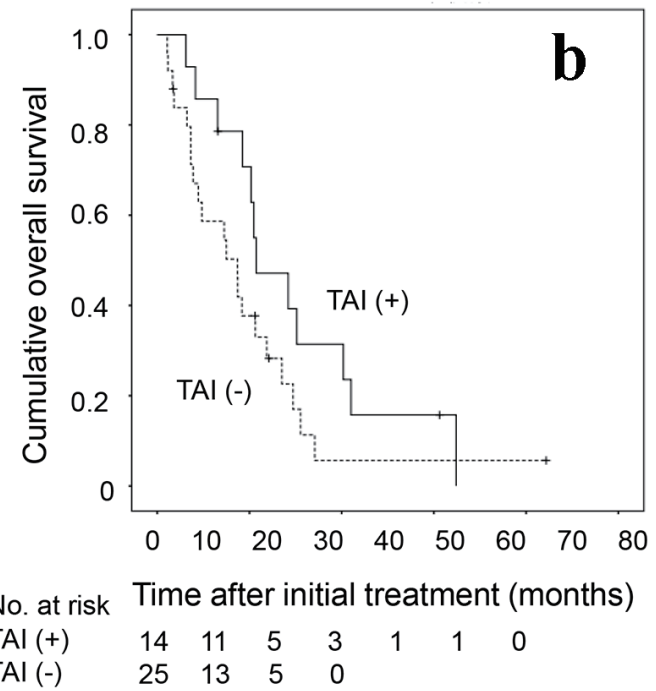

$\begin{array}{llllllll}\mathrm{Al}(+) & 14 & 11 & 5 & 3 & 1 & 1 & 0\end{array}$

Figure 4. Kaplan-Meier analysis of cumulative OS of the $39 \mathrm{HCC}$ patients who underwent TACE as an initial treatment in relation to the presence or absence of (a) repeated TACE as the after-treatment, (b) the TAI as the after-treatment and (c) the administration of sorafenib. OS: overall survival; HCC: hepatocellular carcinoma; TACE: transarterial chemoembolization; TAI: transarterial infusion chemotherapy.

few investigators revealed that, in patients with intermediatestage (BCLC stage B) HCC refractory to TACE, switching to sorafenib administration yielded prolonged overall survival compared with continuing TACE [19-21]. In the present study, a noteworthy finding is that the combined use of TACE/TAI and sorafenib may provide a survival benefit even in our patients with intermediate-stage $\mathrm{HCC}$ having a large number of $\mathrm{HCC}$ nodules; this partially agreed with these previous reports [1921]. In the present study, sorafenib was not administered in 19 patients in whom the prior TACE/TAI was considered to be ineffective, even though they were diagnosed with Child-Pugh class A at baseline. The main reasons for the inability of the sorafenib administration in them were disagreement of the treatment (11 patients), followed by the deterioration of the liver function or general condition (five patients). As for the remaining (three pa- tients), sorafenib was once commenced but stopped shortly by adverse events. When clinical characteristics were investigated in the presence or absence of sorafenib treatment among patients in whom TACE was performed for initial treatment, the serum albumin level was higher in patients receiving the subsequent sorafenib treatment than in those who did not (Supplementary Material 2, www.jocmr.org). The possibility that this may have biased our findings cannot be ruled out.

In conclusion, our findings indicate that $\mathrm{HCC}$ patients with a large number of HCC nodules may be a particular subgroup of the intermediate $\mathrm{HCC}$ revealing the poorer prognosis, and that tumor burden and liver functional reserve may be prognostic factors in this subgroup of HCC. Our results also suggest that, in patients with a large number of HCC nodules, TACE may contribute to the better antitumor efficacy as an 
initial therapy, and that sorafenib administration may be effective, especially in patients who needed repeated TACE/TAI for the tumor control. However, limitations of this study are the retrospective design of the single-center study and the limited number of patients with considerably biased clinical features. Our study shed some light on the treatment, but the optimal treatment strategy has not still been elucidated in patients with a large number of HCC nodules. Very recently, several drugs other than sorafenib have been proven to have efficacy in advanced HCC in the clinical trials [22-26]. Regorafenib [22], lenvatinib [23], cabozantinib [24], ramucirumab [25] and nivolumab [26] have currently been used, or are going to be used in the near future for advanced $\mathrm{HCC}$ in the clinical settings. It is likely that usage of these new drugs as an initial treatment or the after treatment of the prior TACE/TAI would contribute to the improvement of prognosis in patients with a large number of HCC nodules. Further prospective studies with these drugs including a large number of patients would offer better understanding of the optimal treatment for patients with a large number of HCC nodules.

\section{Supplementary Material}

Suppl 1. Clinical Features of the 18 HCC Patients Who Underwent TACE as an Initial Treatment and Their Survival of $>6$ Months and Subsequent TACE or TAI for Twice or More.

Suppl 2. Clinical Features of the 39 HCC Patients With 30 Nodules or More Who Underwent TACE as an Initial Treatment With and Without Subsequent Sorafenib Administration.

\section{Acknowledgments}

None to declare.

\section{Financial Disclosure}

None to declare.

\section{Conflict of Interest}

All authors declare no conflict of interest related to this article.

\section{Informed Consent}

An opt-out approach was used to obtain informed consent from the patients or their family, and personal information was protected during data collection.

\section{Author Contributions}

Takahiro Tabuchi contributed to the statistical analysis. All au- 
thors have made contributions to the conception and design of this study, data collection, and revising the manuscript. All authors approved the final version of manuscript.

\section{References}

1. Ferlay J, Shin HR, Bray F, Forman D, Mathers C, Parkin DM. Estimates of worldwide burden of cancer in 2008: GLOBOCAN 2008. Int J Cancer. 2010;127(12):28932917.

2. Bruix J, Sherman M. Management of hepatocellular carcinoma. Hepatology. 2005;42(5):1208-1236.

3. Omata M, Lesmana LA, Tateishi R, Chen PJ, Lin SM, Yoshida H, Kudo M, et al. Asian Pacific Association for the Study of the Liver consensus recommendations on hepatocellular carcinoma. Hepatol Int. 2010;4(2):439474.

4. Bruix J, Sherman M, American Association for the Study of Liver D. Management of hepatocellular carcinoma: an update. Hepatology. 2011;53(3):1020-1022.

5. European Association For The Study Of The L, European Organisation For R, Treatment Of C. EASL-EORTC clinical practice guidelines: management of hepatocellular carcinoma. J Hepatol. 2012;56(4):908-943.

6. Llovet JM, Bru C, Bruix J. Prognosis of hepatocellular carcinoma: the BCLC staging classification. Semin Liver Dis. 1999;19(3):329-338.

7. Bolondi L, Burroughs A, Dufour JF, Galle PR, Mazzaferro V, Piscaglia F, Raoul JL, et al. Heterogeneity of patients with intermediate (BCLC B) Hepatocellular Carcinoma: proposal for a subclassification to facilitate treatment decisions. Semin Liver Dis. 2012;32(4):348-359.

8. Yamakado K, Miyayama S, Hirota S, Mizunuma K, Nakamura K, Inaba Y, Maeda H, et al. Subgrouping of intermediate-stage (BCLC stage B) hepatocellular carcinoma based on tumor number and size and Child-Pugh grade correlated with prognosis after transarterial chemoembolization. Jpn J Radiol. 2014;32(5):260-265.

9. Kimura H, Ohkawa K, Miyazaki M, Sakakibara M, Imanaka K, Tamura T, Sueyoshi H, et al. Subclassification of patients with intermediate-stage (Barcelona Clinic Liver Cancer stage-B) hepatocellular carcinoma using the up-to-seven criteria and serum tumor markers. Hepatol Int. 2017;11(1):105-114.

10. Weinmann A, Koch S, Sprinzl M, Kloeckner R, SchulzeBergkamen H, Duber C, Lang H, et al. Survival analysis of proposed BCLC-B subgroups in hepatocellular carcinoma patients. Liver Int. 2015;35(2):591-600.

11. Wang JH, Kee KM, Lin CY, Hung CH, Chen CH, Lee CM, $\mathrm{Lu}$ SN. Validation and modification of a proposed substaging system for patients with intermediate hepatocellular carcinoma. J Gastroenterol Hepatol. 2015;30(2):358363.

12. Gehan EA. A generalized wilcoxon test for comparing arbitrarily singly-censored samples. Biometrika. 1965;52:203-223.

13. Takayasu K, Arii S, Ikai I, Omata M, Okita K, Ichida T, Matsuyama Y, et al. Prospective cohort study of tran- sarterial chemoembolization for unresectable hepatocellular carcinoma in 8510 patients. Gastroenterology. 2006;131(2):461-469.

14. Shiina S, Tateishi R, Arano T, Uchino K, Enooku K, Nakagawa H, Asaoka Y, et al. Radiofrequency ablation for hepatocellular carcinoma: 10-year outcome and prognostic factors. Am J Gastroenterol. 2012;107(4):569-577; quiz 578.

15. Wilhelm SM, Carter C, Tang L, Wilkie D, McNabola A, Rong H, Chen C, et al. BAY 43-9006 exhibits broad spectrum oral antitumor activity and targets the RAF/MEK/ ERK pathway and receptor tyrosine kinases involved in tumor progression and angiogenesis. Cancer Res. 2004;64(19):7099-7109.

16. Llovet JM, Ricci S, Mazzaferro V, Hilgard P, Gane E, Blanc JF, de Oliveira AC, et al. Sorafenib in advanced hepatocellular carcinoma. N Engl J Med. 2008;359(4):378-390.

17. Cheng AL, Kang YK, Chen Z, Tsao CJ, Qin S, Kim JS, Luo R, et al. Efficacy and safety of sorafenib in patients in the Asia-Pacific region with advanced hepatocellular carcinoma: a phase III randomised, double-blind, placebocontrolled trial. Lancet Oncol. 2009;10(1):25-34.

18. Iavarone $\mathrm{M}$, Cabibbo $\mathrm{G}$, Piscaglia F, Zavaglia C, Grieco A, Villa E, Camma C, et al. Field-practice study of sorafenib therapy for hepatocellular carcinoma: a prospective multicenter study in Italy. Hepatology. 2011;54(6):20552063.

19. Ogasawara S, Chiba T, Ooka Y, Kanogawa N, Motoyama T, Suzuki E, Tawada A, et al. Efficacy of sorafenib in intermediate-stage hepatocellular carcinoma patients refractory to transarterial chemoembolization. Oncology. 2014;87(6):330-341.

20. Arizumi T, Ueshima K, Chishina H, Kono M, Takita M, Kitai S, Inoue T, et al. Validation of the criteria of transcatheter arterial chemoembolization failure or refractoriness in patients with advanced hepatocellular carcinoma proposed by the LCSGJ. Oncology. 2014;87(Suppl 1):3236.

21. Ohki T, Sato K, Yamagami M, Ito D, Yamada T, Kawanishi K, Kojima K, et al. Efficacy of transcatheter arterial chemoembolization followed by sorafenib for intermediate/advanced hepatocellular carcinoma in patients in Japan: a retrospective analysis. Clin Drug Investig. 2015;35(11):751-759.

22. Bruix J, Qin S, Merle P, Granito A, Huang YH, Bodoky G, Pracht M, et al. Regorafenib for patients with hepatocellular carcinoma who progressed on sorafenib treatment (RESORCE): a randomised, double-blind, placebocontrolled, phase 3 trial. Lancet. 2017;389(10064):56-66.

23. Kudo M, Finn RS, Qin S, Han KH, Ikeda K, Piscaglia F, Baron A, et al. Lenvatinib versus sorafenib in first-line treatment of patients with unresectable hepatocellular carcinoma: a randomised phase 3 non-inferiority trial. Lancet. 2018;391(10126):1163-1173.

24. Abou-Alfa GK, Meyer T, Cheng AL, El-Khoueiry AB, Rimassa L, Ryoo BY, Cicin I, et al. Cabozantinib in patients with advanced and progressing hepatocellular carcinoma. N Engl J Med. 2018;379(1):54-63.

25. Zhu AX, Kang YK, Yen CJ, Finn RS, Galle PR, Llovet 
JM, Assenat E, et al. Ramucirumab after sorafenib in patients with advanced hepatocellular carcinoma and increased alpha-fetoprotein concentrations (REACH-2): a randomised, double-blind, placebo-controlled, phase 3 trial. Lancet Oncol. 2019;20(2):282-296.
26. El-Khoueiry AB, Sangro B, Yau T, Crocenzi TS, Kudo M, Hsu C, Kim TY, et al. Nivolumab in patients with advanced hepatocellular carcinoma (CheckMate 040): an open-label, non-comparative, phase 1/2 dose escalation and expansion trial. Lancet. 2017;389(10088):2492-2502. 